\title{
Sonographically Guided Lumbar Puncture in Pediatric Patients
}

Page I. Wang, MD, Anthony C. Wang, MD, Jennifer O. Naidu, MD, Michael A. DiPietro, MD, Pia C. Sundgren, MD, PhD, Douglas J. Quint, MD, Hemant A. Parmar, MD, Gaurang V. Shah, MD, Karin M. Muraszko, MD, Ashok Srinivasan, MD

Received February 5, 2013, from the Departments of Radiology (P.I.W., M.A.D., P.C.S., D.J.Q. H.A.P., G.V.S., A.S.), Neurosurgery (A.C.W., K.M.M.), and Pediatrics (J.O.N.), University of Michigan Hospital, Ann Arbor, Michigan USA; and Department of Radiology, Institute for Clinical Sciences, Lund University, Lund, Sweden (P.C.S.). Revision requested February 25, 2013. Revised manuscript accepted for publication April 22, 2013.

We thank Rita Ayyangar, MD, Margy Fox, PAC, Hugh Garton, MD, Elaine Pomeranz, MD, and Michelle Kleinhenz, NP, for helping recruit patients and Leslie Burrell for illustrations. This work was supported by a University of Michigan Fostering Innovations grant and a University of Michigan/Department of Neurosurgery Motor City Golf Classic grant.

Address correspondence to Page I. Wang, MD, Department of Radiology, University of Michigan, A. Alfred Taubman Health Care Center, 1500 E Medical Center Dr, Ann Arbor, MI 48109 USA.

E-mail:pagew@umich.edu

\section{Abbreviations}

ALL, acute lymphoblastic leukemia; BMI, body mass index; CSF, cerebrospinal fluid; $R B C$, red blood cell

doi:10.7863/ultra.32.12.2191
Our aim with this study was to develop a user-friendly method for pediatric sonographically guided lumbar punctures so that we can visualize intrathecal anatomy, confirm intrathecal injection at the time of injection, and, most importantly, avoid ionizing radiation to a child's already radiosensitive pelvis. Sonographically guided lumbar puncture was prospectively performed in children aged 7 weeks to 16 years. All attempts $(n=9)$ were successful. We were able to identify relevant anatomy (including the conus in children 10 years and younger), confirm intrathecal injection, visualize intrathecal hematoma, and avoid radiation. Sonography is a promising modality for image-guided lumbar punctures without radiation in children.

Key Words_-lumbar puncture; pediatric; sonography

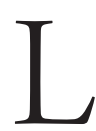
umbar puncture is a procedure performed in infants and children to obtain cerebrospinal fluid (CSF). Traditionally, when an unguided lumbar puncture fails, a fluoroscopically guided lumbar puncture is ordered. Although fluoroscopically guided lumbar punctures have been shown to decrease the rate of traumatic taps compared to unguided lumbar punctures, there are several reasons why fluoroscopic guidance is suboptimal, especially in children. ${ }^{1}$ Fluoroscopic guidance confers radiation, which is less helpful in infants with nonossified posterior elements and does not allow for visualization of the intrathecal space containing the conus or occlusive hematoma. Visualization of the intrathecal space would be helpful, as a recent study found that $58.8 \%$ of fluoroscopically guided lumbar punctures fail in infants, presumably from intrathecal hematoma occluding the thecal sac. ${ }^{2,3}$

Sonographically guided lumbar puncture is ideal for infants and children because it is nonionizing, allows for visualization of the intrathecal space (in some children), and can confirm the location of medication, such as chemotherapy. Sonographically guided lumbar puncture has been shown to be useful in the obstetric, emergency, and neonatal settings, but to our knowledge, no study has looked at children older than 1 year. ${ }^{4-7}$

Our aim with this study was to develop a user-friendly sonographically guided lumbar puncture method for all pediatric patients, so that we can visualize intrathecal anatomy, confirm intrathecal injection at the time of injection, and, most importantly, avoid ionizing radiation to a child's already radiosensitive pelvis. 


\section{Materials and Methods}

After Institutional Review Board approval was obtained for this prospective study, pediatric patients referred to radiology for fluoroscopically guided lumbar puncture were recruited for sonographically guided lumbar puncture from September 2011 to September 2012. Informed written consent was obtained from each patient or the parent. Patients between the ages of 0 and 18 years were eligible for enrollment.

All sonographically guided lumbar punctures were performed by 2 investigators (A.S. and G.V.S., who are board-certified neuroradiologists; 8 of 9 were performed by A.S.). All patients were placed in the left lateral decubitus position or prone with lumbar spine flexion to open the interspinous space. Curved ultrasound transducers were used to find the conus medullaris by angling the transducer toward the midthecal sac (Figure 1). If the conus was seen, the level was marked on the patient's skin. If the conus was not seen, a transverse line (Tuffier line) was also marked across the patient's palpated superior-inferior iliac crests to indicate an appropriate level for lumbar puncture. Once an appropriate level for lumbar puncture was marked (either below the conus or along the Tuffier line), sonography in the longitudinal plane was used to identify the spinous processes and lamina (Figure 2). Holding the transducer in a longitudinal orientation, the midline spinous process line and paramidline lamina were marked with vertical lines, and the intraspinous space was marked with a horizontal line (Figure 3). A location between the horizontal laminar line and spinous process lines along the intraspinous line was marked with an X (Figure 3). The patient and the ultrasound transducer were then sterilely prepared, the patient was draped, and $1 \%$ lidocaine was subcutaneously injected into the area marked with an X. The ultrasound transducer was placed in a transverse orientation at the level of the $\mathrm{X}$, and a 22-gauge spinal needle was inserted with slight medial angulation toward the midline vertical spinous process line using a freehand technique. If intrathecal medication was injected, color Doppler sonography was used during the injection to confirm the location of the medication. The number of attempts was

Figure 1. Longitudinal parasagittal spinal sonograms from a 10-year-old male patient. A, Paramidline sagittal image taken at approximately the T12 level showing a hypoechoic tapering conus (left brace), dura (arrowhead), lamina (arrows), and vertebral body (star). B, If the transducer is directed laterally at the level of the conus, the cauda equina nerve roots will be visible (right brace).

A

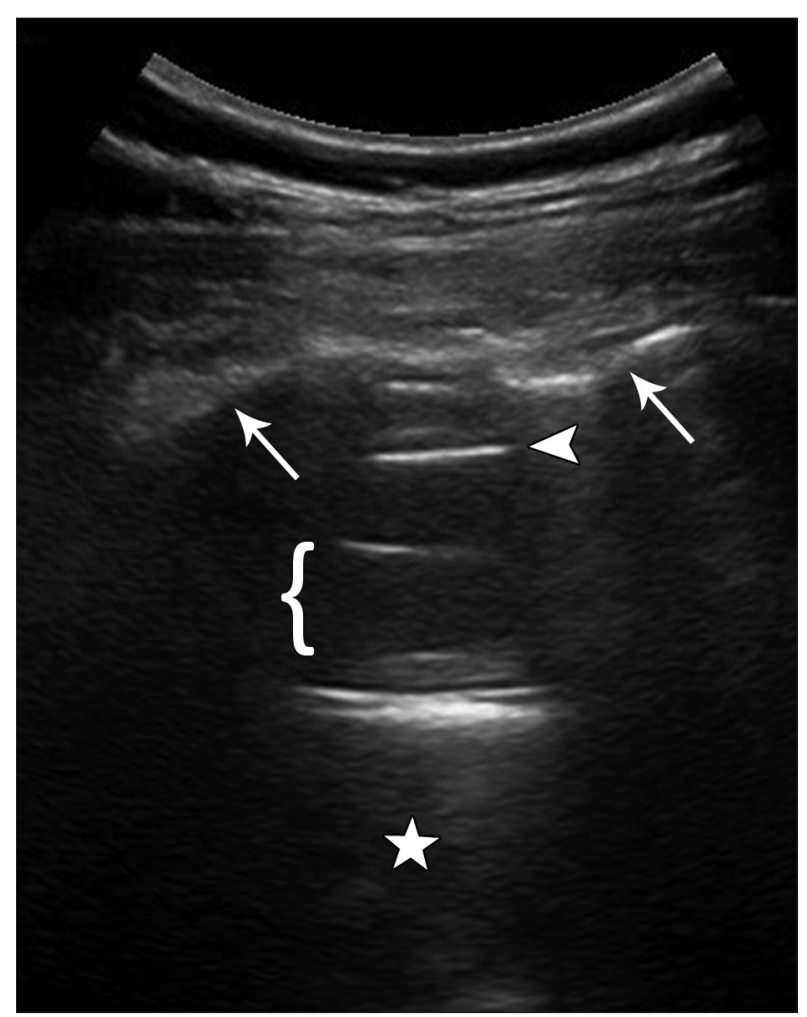

B

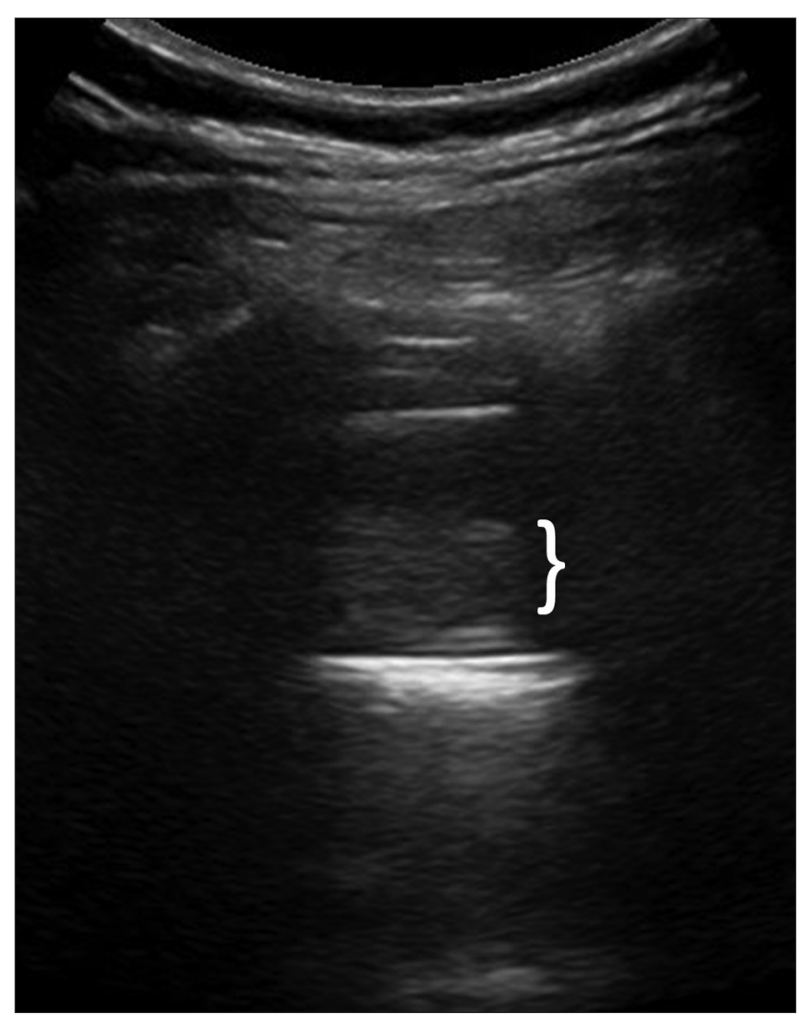


defined as the number of cutaneous punctures made by the spinal needle. Success was defined as the return of CSF from the spinal needle after the intrathecal space was reached, and a traumatic tap was defined as more than 500 red blood cells (RBCs) $/ \mathrm{mm}^{3}$ in the first vial of CSF. ${ }^{8}$

Of the 9 patients, 4 received general anesthesia, 4 received conscious sedation, and 1 had local anesthesia only. Two patients were already under general anesthesia for other procedures.

\section{Results}

A total of 9 patients were included in the study, ranging in age from 7 weeks to 16 years. Patient demographics are summarized in Table 1. A summary of the curved ultrasound transducer choices, numbers of attempts, and CSF findings can be seen in Table 2. Several transducers were used from different machines, but none was found to be consistently superior to the others.

We found that the osseous landmarks were quickly identified by sonography, even in an obese child with a body mass index (BMI) of $32 \mathrm{~kg} / \mathrm{m}^{2}$. The spinal cord was readily visualized in children up to the age of 10 years but not well seen in children older than 10 years. Sonography allowed us to visualize and track the needle path from the subcutaneous tissues to the level of the thecal sac. If a patient needed intrathecal medication, we could confirm the intrathecal location by Doppler signal/pseudoflow artifact, which was not present before but was present after the injection (Figure 4). ${ }^{9}$

A

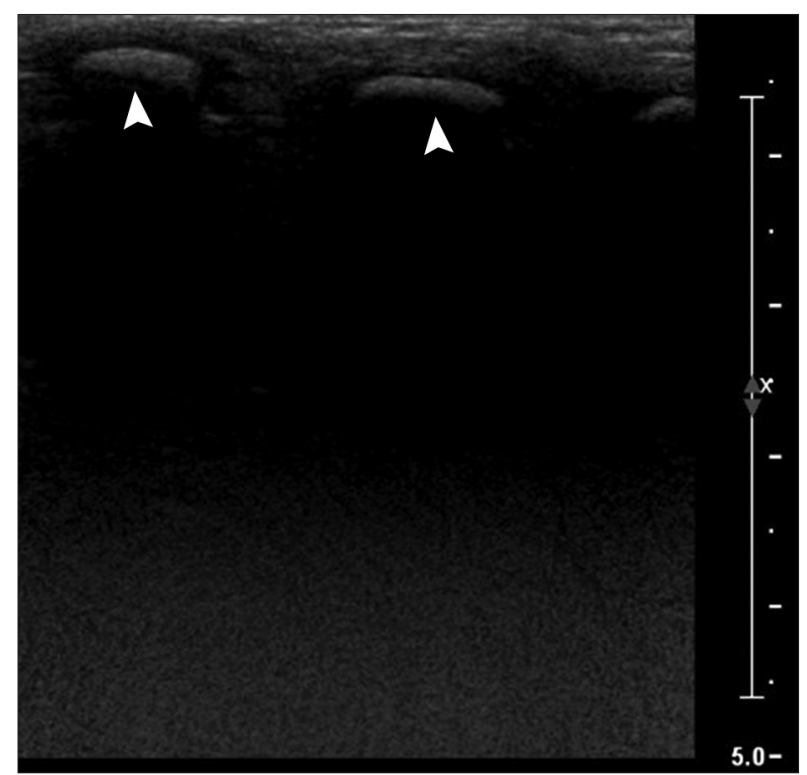

One of our adult-sized 16-year-old patients with acute lymphoblastic leukemia (ALL) had several failed unguided attempts before the ordering of image-guided lumbar puncture. We were able to reach the CSF in a single attempt with only $1 \mathrm{RBC} / \mathrm{mm}^{3}$ in the CSF.

Another patient who was 7 weeks old had 6 failed prior attempts, and the intrathecal space surrounding the spinal cord was echogenic (most likely from hemorrhage), but we found a smaller pocket of hypoechoic fluid by the cauda equina and directed our needle toward that CSF collection (Figure 5). This patient did have more than 500 $\mathrm{RBCs} / \mathrm{mm}^{3}$ in the collected CSF but did have multiple prior attempts.

Sonographically guided lumbar puncture was successful in all patients. No adverse events occurred during or immediately after any procedure.

\section{Discussion}

Fluoroscopy is used as the primary mode of image guidance for lumbar puncture in many radiology departments in the United States. However, there are several disadvantages of this technique in the pediatric population. First, children are exposed to ionizing radiation. Although the consequences have yet to be adequately studied, prudent clinical practice is to limit radiation exposure whenever

Figure 2. Longitudinal sagittal and parasagittal spinal sonograms from a 5-year-old male patient. A, Sagittal image showing the superficially located inverted-U appearance of the spinous processes (arrowheads). B, Parasagittal image showing the sloping lamina (arrows), which should not be mistaken for the spinous processes.

B

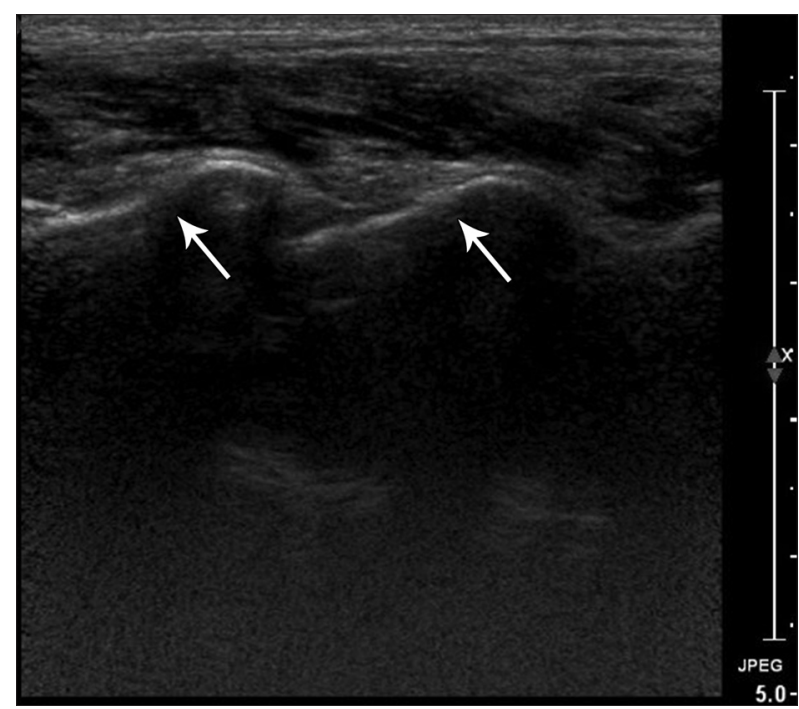


possible. Second, fluoroscopically guided lumbar punctures are frequently performed in the prone position, which decreases spinal flexion and at our institution often necessitates endotracheal intubation and general anesthesia for airway control in children. Third, fluoroscopically guided lumbar punctures cannot confirm intrathecal injection of medication at the time of injection without the addition of contrast material. Last, fluoroscopy does not allow for visualization of the thecal space, so a low-lying conus or occlusive thecal hematoma would be invisible.

Using sonographic guidance, we were able to successfully access the intrathecal space in children aged 7 weeks to 16 years with BMIs up to $32 \mathrm{~kg} / \mathrm{m}^{2}$ without radiation. Instead of our usual prone positioning for fluoroscopically guided lumbar puncture, we positioned patients in the lateral decubitus position, which allowed a greater degree of lumbar spine flexion. Doppler aliasing allowed confirmation of intrathecal injection of medication at the time of injection, which is important when administrating intrathecal medication. One of the patients had ALL, and we were able to nontraumatically reach the CSF with a single attempt, which is important, as some data suggest that traumatic taps in patients with ALL portend a worse prognosis. ${ }^{10}$ Last, the conus medullaris was visualized in children up to 10 years and confidently avoided (Figure 1).

The method that we found most helpful was to draw vertical lines to landmark the spinous processes and lamina and a horizontal line denoting the intraspinous space. We used a parasagittal approach with the needle between the spinous process and lamina; other authors have used a midline approach, marked the anatomy with sonography, and blindly directed the needle through the intraspinous space. ${ }^{11,12}$ We did use real-time needle guidance for our method, but other operators may find that using sonography to indicate landmarks and proceeding without realtime needle guidance may be more user friendly.

Figure 3. Ultrasound transducer positioning for real-time needle guidance. A, Prone view of the spine. The spine is marked below the conus and/or at the level of the superior-inferior iliac crests. Sonography in the longitudinal plane is used to identify the lamina, spinous processes, and intraspinous space, which are marked on the patient by vertical and horizontal lines drawn on the patient's back (see dotted lines). An X is marked between a vertical laminar line and the midline spinous processes along the intraspinous line. The position of the transducer is outlined by a box. B, Axial view of the spine. After the patient is prepared and draped, a sterile ultrasound transducer is positioned adjacent to the X, which has been numbed with lidocaine, and the needle is directed medially toward the thecal sac.

A

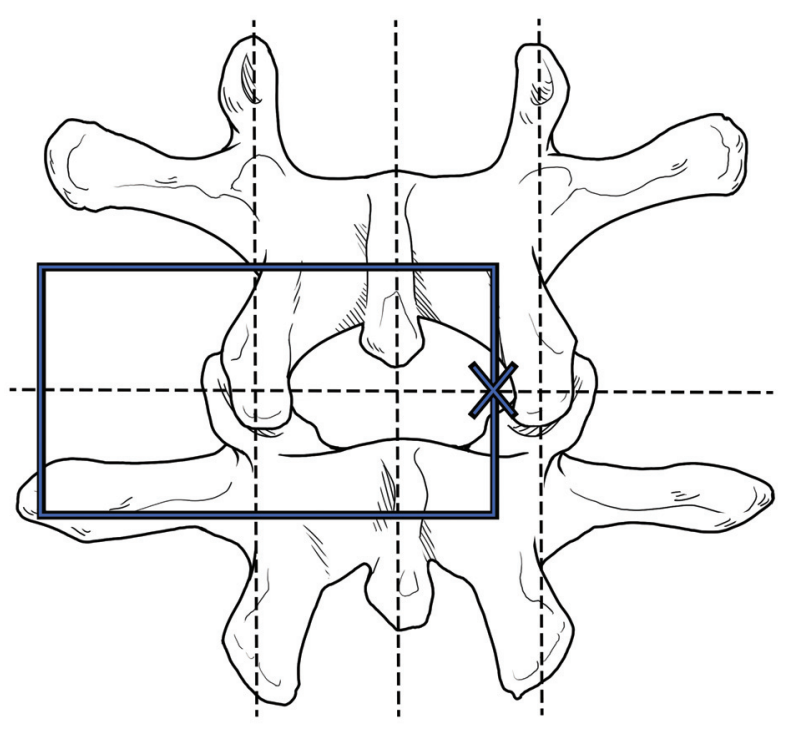

B

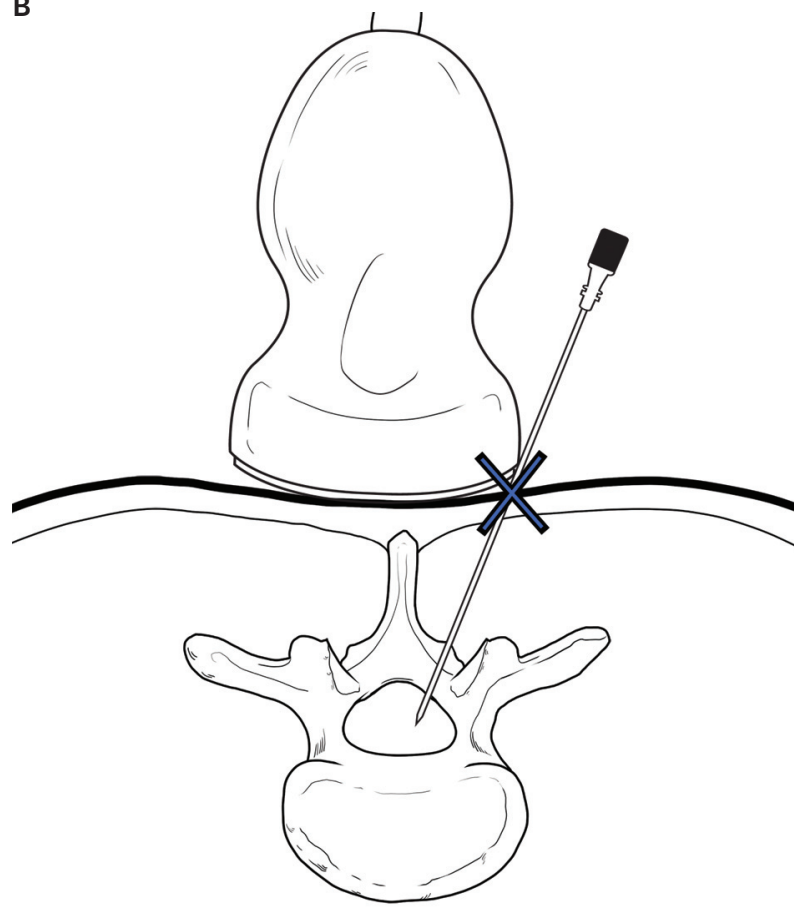


Table 1. Patient Demographics

\begin{tabular}{llccl}
\hline Age & Sex & Weight, $\mathbf{k g}$ & BMI, $\mathbf{k g} / \mathbf{m}^{\mathbf{2}}$ & \multicolumn{1}{c}{ Reason for Lumbar Puncture/Comorbidity } \\
\hline $12 \mathrm{y}$ & Male & 20.8 & 15.5 & Cerebral palsy, baclofen injected \\
$7 \mathrm{mo}$ & Male & 6.7 & 17.1 & Myelogram for nasoethmoidal encephalocele \\
$10 \mathrm{y}$ & Male & 36.5 & 19.1 & Cerebral palsy, baclofen injected \\
$14 \mathrm{y}$ & Male & 31.0 & 17.8 & Cerebral palsy, rule out infection, CSF collected \\
$9 \mathrm{y}$ & Female & 32.1 & 17.6 & Cerebral palsy, baclofen injected \\
$16 \mathrm{y}$ & Female & 65.9 & 25.7 & Headaches, rule out idiopathic intracranial hypertension, \\
& & & & CSF collected \\
$5 \mathrm{y}$ & Male & 13.8 & 14.2 & Cerebral palsy, baclofen injected \\
$15 \mathrm{y}$ & Female & 88.3 & 32.0 & ALL, CSF collected, intrathecal chemotherapy \\
$7 \mathrm{wk}$ & Female & 5.1 & 6.8 & Concern for herpes simplex virus, seizure \\
\hline
\end{tabular}

Table 2. Summary of Lumbar Puncture Procedures

\begin{tabular}{llccc}
\hline Age & Transducer & Attempts & $\begin{array}{c}\text { CSF Collected } \\
\text { and Sent to Lab? }\end{array}$ & $\begin{array}{c}\text { RBCs/mm } \\
\text { in First Vial }\end{array}$ \\
\hline $12 \mathrm{y}$ & Philips C8-5 & 1 & No & NA \\
$7 \mathrm{mo}$ & Zonare C9-4 & 1 & No & NA \\
$10 \mathrm{y}$ & Zonare C9-3 & 1 & No & 162 \\
$14 \mathrm{y}$ & Philips C8-5 & 3 & Yes & NA \\
$9 \mathrm{y}$ & Philips C9-5 & 1 & No & 1 \\
$16 \mathrm{y}$ & Zonare C4-1 & 1 & Yes & NA \\
$5 \mathrm{y}$ & Philips C12-9 & 2 & Yes & 1 \\
$15 \mathrm{y}$ & Philips C5-1 & 1 & Yes & 4440 \\
$7 \mathrm{wk}$ & Philips C8-5 & 1 & & \\
\hline
\end{tabular}

NA indicates not applicable.

aPhilips Healthcare (Bothell, WA); Zonare Medical Systems (Mountain View, CA).

Figure 4. Transverse spinal sonograms from a 10-year-old male patient. A, Color Doppler image showing a pseudoflow artifact within the thecal sac, confirming intrathecal injection of baclofen. B, Image using the same color Doppler settings from the same patient without injection of baclofen.

A

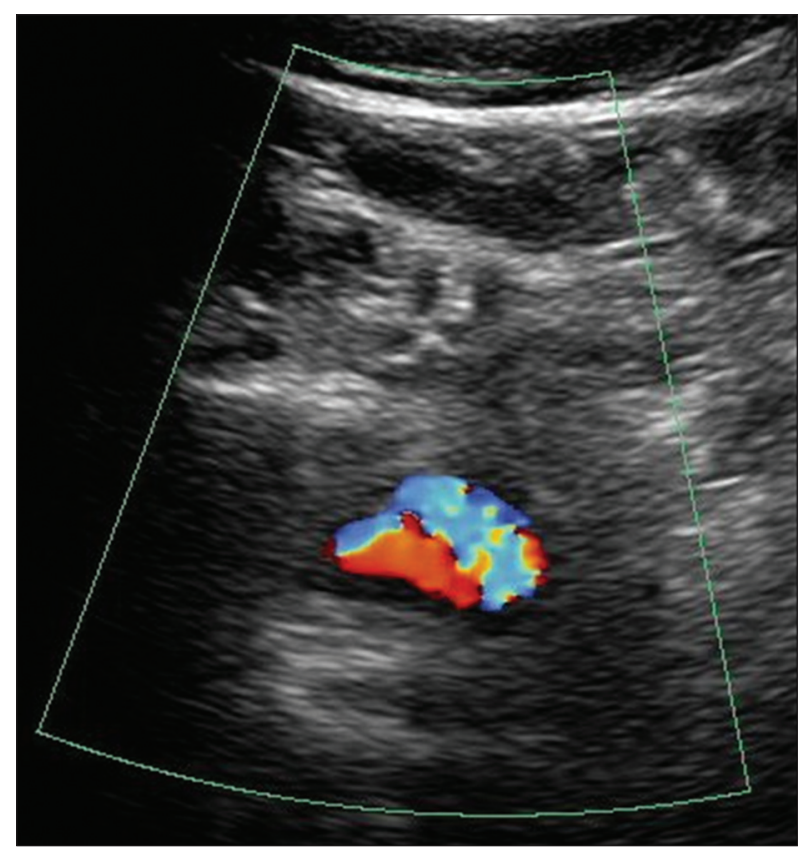

B

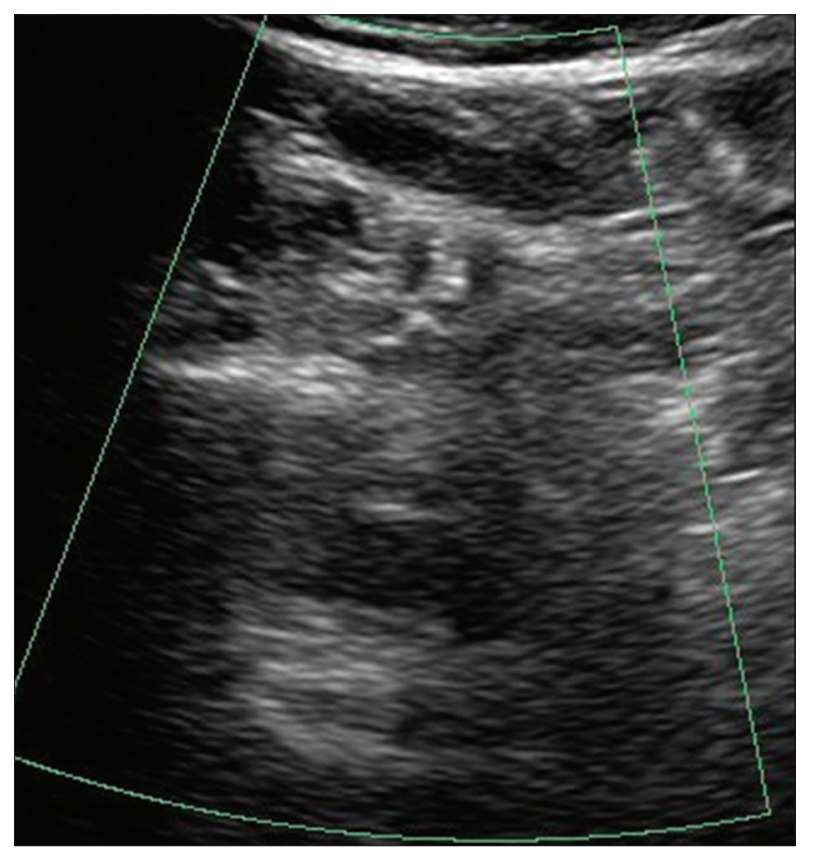




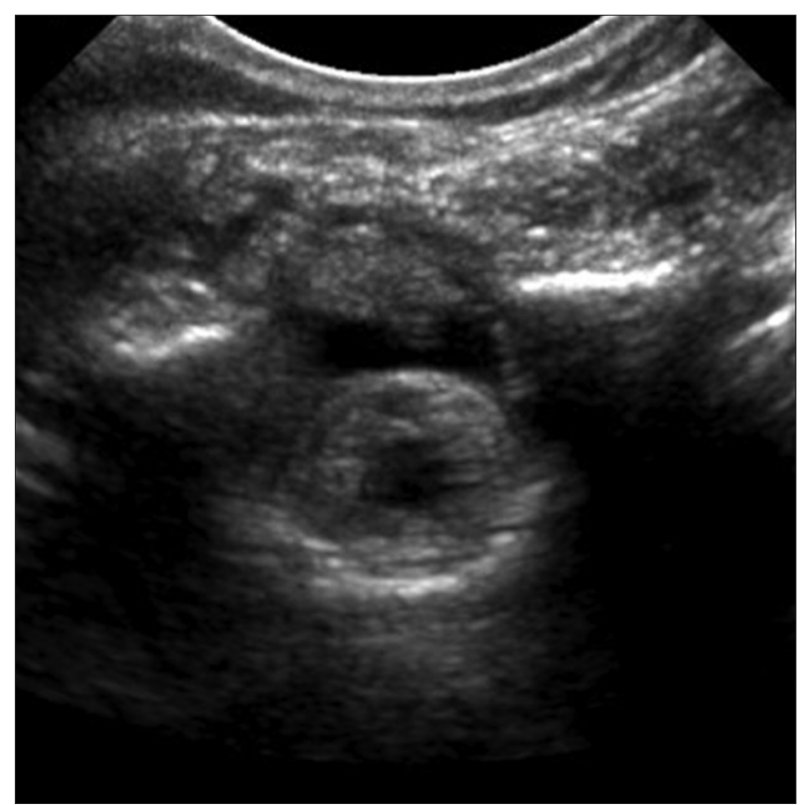

A

Figure 5. Transverse sonograms from a 7-week-old female patient after multiple failed lumbar puncture attempts. A, Image at the level of the thoracic

spinal cord showing surrounding echogenic material. B, More caudally, at the level of the cauda equina, most of the echogenic material is replaced by a hypoechoic fluid collection (arrow).

The main limitation of this study was the small sample size because of the limited referrals for pediatric lumbar puncture at our institution, likely from the high rate of successful nonguided lumbar punctures in children. However, even in this limited sample, we achieved a $100 \%$ success rate with sonographically guided lumbar punctures. Another limitation was that when the conus medullaris could not be visualized, the vertebral body level was estimated by the superior-inferior iliac crests, which has been shown to be inconsistent. ${ }^{4}$ Additionally, we did find it difficult to use sonography on the patient with rotary scoliosis (3 attempts), secondary to an awkward needle angle. Most of our patients were under general or conscious sedation, and the decreased patient motion likely made it easier to achieve nontraumatic taps.

In terms of future directions, we plan on enrolling more patients (including adults) in the hope that sonography will become the first-line modality for image-guided lumbar puncture. We will also continue to gather CSF so that we can more directly compare the rates of traumatic taps in sonographically guided lumbar punctures to those in fluoroscopically guided lumbar punctures.

In sum, real-time sonographically guided lumbar puncture is a promising technique, which confers many advantages compared to unguided and fluoroscopically guided lumbar puncture techniques, especially in children.

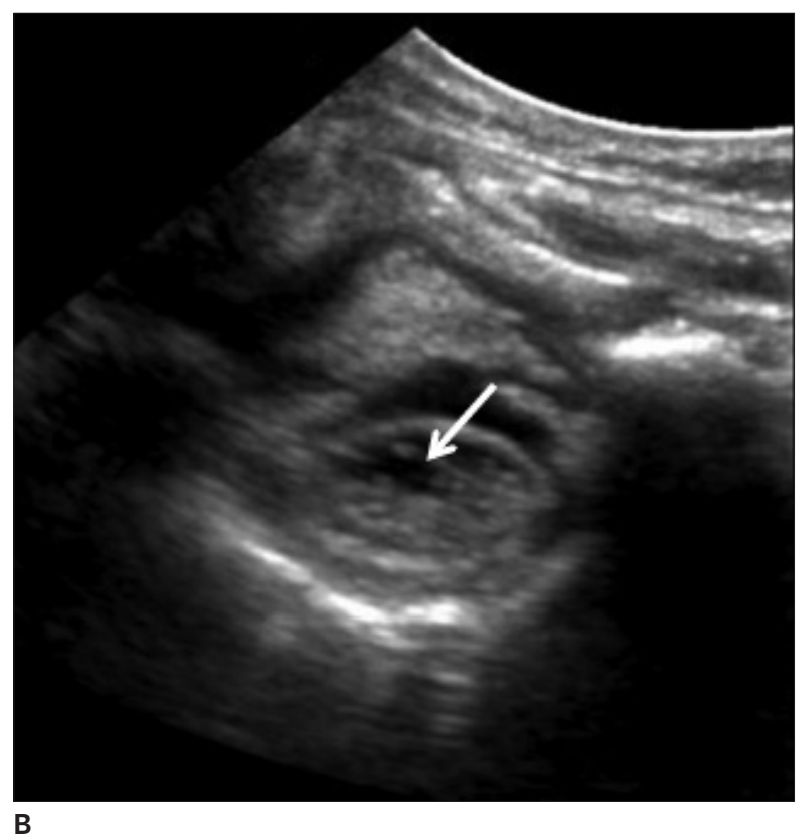


9. Campbell SC, Cullinan JA, Rubens DJ. Slow flow or no flow? Color and power Doppler US pitfalls in the abdomen and pelvis. Radiographics 2004; 24:497-506.

10. Gajjar A, Harrison PL, Sandlund JT, et al. Traumatic lumbar puncture at diagnosis adversely affects outcome in childhood acute lymphoblastic leukemia. Blood 2000; 96:3381-3384.

11. Ayalin T, Lam SH. Ultrasound-assisted lumbar puncture in infants. Acad Emerg Med 2011; 18:e36.

12. Chin KJ, Perlas A, Chan V, Brown-Shreves D, Koshkin A, Vaishnav V. Ultrasound imaging facilitates spinal anesthesia in adults with difficult surface anatomic landmarks. Anesthesiology 2011; 115:94-101. 